\title{
$U B V$ absolute CCD photometry and differential astrometry of a sample of visual double stars with A-type primaries ${ }^{\star, \star \star}$
}

\author{
N. Shatsky ${ }^{1,2}$, D. Sinachopoulos ${ }^{1}$, P. Prado ${ }^{3}$, and E. van Dessel ${ }^{1}$ \\ 1 Royal Belgian Observatory, Ringlaan 3, B-1180 Brussels, Belgium \\ 2 Sternberg Astronomical Institute, Universitetskii pr. 13, Moscow 119899, Russia \\ 3 European Southern Observatory, La Silla, Chile
}

Received January 4; accepted May 5, 1999

\begin{abstract}
We present $V$ magnitudes, $(B-V)$ and $(U-B)$ colours of the components of 111 visual double stars and their relative position measurements $\rho$ and $\theta$. The primaries are of spectral class $\mathrm{A}$; the angular separation is in the $2^{\prime \prime} .5-8^{\prime \prime}$ range. The observations were made at the $61-\mathrm{cm}$ reflector of University of Toronto Southern Observatory, Chile. The obtained photometric results are of a moderate quality for absolute values $\left(0.04^{\mathrm{m}}(\mathrm{mag})\right.$ in $V$ and about $0.02^{\mathrm{m}}$ in the $(B-V)$ and $(U-B)$ colour indices) and more precise in $\Delta V$ and $\Delta(U-B), \Delta(B-V)$ $\left(0.023^{\mathrm{m}}\right.$ and $0.015^{\mathrm{m}}$, respectively $)$. We find that about $60 \%$ of secondaries are also of spectral type A; $56 \%$ of pairs have $\Delta(B-V)$ less than $0.1^{\mathrm{m}}$. The two-colour diagram reveals significant offset of objects from the curve of unreddened Main Sequence stars. A comprehensive survey of stars in the close vicinity to the objects is also presented. The final astrometric accuracy of $\approx 00^{\prime \prime} .01$ in $\rho$ and 0.1 in $\theta$ provides additional measurements for study of relative motion of the components.
\end{abstract}

Key words: stars: binaries: visual - techniques: photometric - astrometry

\section{Introduction}

The investigation of binary stars is a key to many problems of modern astrophysics. Visual double stars, which represent the tail of longest orbital periods in the distribution of binaries in general, are in fact poorly studied

Send offprint requests to: N. Shatsky

* Based on observations made at University of Toronto Southern Observatory, Las Campanas, Chile.

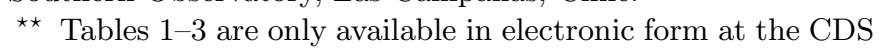
via anonymous ftp to cdsarc.u-strasbg.fr (130.79.128.5) or via http://cdsweb.u-strasbg.fr/Abstract.html

Correspondence to: kolja@sai.msu.su so far. Although they have been observed for about two centuries, their physics and, in most cases of wide pairs, even the question of "opticity" of the companions are still under discussion.

This work is a part of the extended investigation of visual double stars with primaries of spectral class $A$. The aim is to obtain reliable statistics of the photometric properties of their components. These data are required in the frame of determination of their evolutionary status and possible mechanisms of formation. Investigation of newly obtained precise astrometric information may also help to reject optical pairs by comparison of their relative motion with the possible orbital one (Russel \& Moore 1940).

For this purpose we selected the binaries of class A from the Washington Double Stars catalogue (Worley \& Douglass 1984, hereafter WDS), which have separations in the range from 2 to 8 arcsec. These limits were set by the resolution capabilities of the available observational technique from one point and by the desire to have a moderately low rate of optical pairs from the other. In general there is no photometric information on primary and secondary components separately and no recent astrometry. So, the new data will improve our knowledge of the physical nature of these moderately young wide binaries.

\section{Observations and primary treatment of images}

In 1991-92 more than one hundred doubles were observed at Las Campanas observatory (Chile) by P. P.. The evening dates of nights of observations were I. 5 May; II. 17, 18 Sep.; III. 30 Nov., 10 Dec. 1991; IV. 16-18, 20-24 Feb.; V. 15-17, 19 Mar. 1992 (the missions are denoted by roman letters). The mean epoch is 1992.0.

A nitrogen cooled CCD camera was attached to the 61-cm Cassegrain telescope. The chip had the dimensions $512 \times 512$ with square pixels and low readout noise of 5 electrons. During the observations only the central $258 \times 258$ pixels window was read. 
The camera was equipped with a set of wide band filters which approximately corresponded to the $U, B$ and $V$-bands of the Johnson photometric system. Some objects were observed in $B$ and $V$ only. Flat fields of the dusk sky or dome in all filters and bias frames were taken each night before observations. For the targets about 3 frames were exposed in $V, 4-6$ in $B$ and $8-10$ in $U$ bands. Exposure duration varied from $3 \mathrm{~s}$ in $V$ to $30 \mathrm{~s}$ in $U$. More than half of the objects were observed twice in different nights.

To obtain the absolute photometry data on our objects observations were restricted to photometric nights. The information on extinction was planned to be taken afterwards from Geneva data (see Burki et al. 1995) obtained at nearby La Silla. To determine the transformations from instrumental to standard magnitudes one night in the end of November 1991 was devoted to standard star observations.

In order to calibrate the astrometric parameters of our objects some astrometric standard binaries with well established positional parameters were observed in the $V$-filter to give us the scale and orientation of the detector. They were selected from the list of Brosche \& Sinachopoulos (1988) created in the frame of the Hipparcos project. Several stellar traces obtained with the stopped telescope clock drive were also made to determine independently the orientation of chip.

Observational conditions proved to be satisfactory; the seeing was mostly below $1^{\prime \prime}-1$.' 5 . The transparency variations were quite low with few exceptions (see below Table 2). The ambient temperature changed from one observational session to another in the range from $9^{\circ} \mathrm{C}$ to $18^{\circ} \mathrm{C}$.

All in all more than 2600 frames were taken. The frames were reduced (removal of cosmic ray hits, bias subtraction and flat-field division) using the ESO-MIDAS software package. The absolute photometry measurements were made by integration of the total object flux in an $\approx 34^{\prime \prime}$ round aperture. Differential parameters of double stars $(\rho, \theta, \Delta \mathrm{Mag})$ were obtained using the LevenbergMarquardt least square fit of the object profile (Press et al. 1987). The two-dimensional Moffat function with an ellipticity in an arbitrary direction was fitted simultaneously to both star images:

$$
\begin{gathered}
\operatorname{Moffat}(x, y)= \\
H
\end{gathered}
$$$$
\overline{\left\{1+B\left[\left(x-X_{0}\right)^{2}+(1+E)\left(y-Y_{0}\right)^{2}+F\left(x-X_{0}\right)\left(y-Y_{0}\right)\right]\right\}^{Q}} .
$$

For wide astrometric standard measurements we used the DAOPHOT package which was checked to give the same results as the Moffat profile fitting.

\section{Photometric reduction}

One basic assumption to obtain the absolute photometry data was that the extinction at cerro Las Campanas is the same as that measured at the Swiss telescope at cerro
La Silla ${ }^{1}$ (which lies only $30 \mathrm{~km}$ to the south). In order to check this we obtained Bouguer curves for an E3-area standard star HD 49789 (Graham 1982) in the night of 30.11/1.12.91. Resulting extinction coefficients were quite close to the values that Genevian data for that night supply:

$$
\begin{aligned}
& k_{v}=0.18 \pm 0.01, \quad k_{v}^{\text {gen }}=0.194 \pm 0.005 \\
& k_{b}=0.31 \pm 0.01, \quad k_{b}^{\text {gen }}=0.310 \pm 0.004 \\
& k_{u}=0.53 \pm 0.01, \quad k_{u}^{\text {gen }}=0.655 \pm 0.009 .
\end{aligned}
$$

One can thus see that there is no significant difference neither in $V$ nor in $B$ filters. The lower extinction in our $U$ filter is interpreted as caused by the slightly "redder" effective wavelength of our filter compared to the Genevian one. Hence, it was decided to apply Genevian values in $B$ and $V$ bands and $80 \%$ of $k_{u}^{\text {gen }}$ value for the extinction correction. The second-order extinction was neglected for two reasons. First, the colours $(B-V)$ of our objects are normally close to zero $(76 \%$ of the components have $(B-V)<0.3)$ and airmass was low $(<1.5)$, so the expected error in $k_{b}, k_{u}$ was estimated not to exceed 0.005 . Second, to account properly for secondary extinction for early-type stars their two-dimensional spectral types have to be known (see Straižys 1972, p. 128).

From 13 photometric standard star measurements we determined the coefficients $C$ and $A$ of linear transformation from the instrumental to the standard $U B V$ system: $M^{\text {std }}-M^{\text {inst }}=C_{M}+A_{M} \times C I^{\text {inst }}$ (here the colour index $C I$ is $(B-V)$ for $M \equiv V,(B-V)$ and $(U-B)$ for $M \equiv$ $(U-B))$. For our standards we used the data of Landolt (1973); Graham (1982) and the improved photometric values kindly provided by Grenon (1992). The derived colour dependency coefficients are: $A_{V}=0.122 \pm 0.005$, $A_{B-V}=0.007 \pm 0.004$ and $A_{U-B}=0.077 \pm 0.014$.

The comparison of the photometric results obtained in different nights allowed us to determine the overall measure of photometry data reliability:

- Absolute photometry standard deviations:

in $V: 0.04^{\mathrm{m}}$, in $(U-B)$ and $(B-V): 0.02^{\mathrm{m}}$;

- Differential photometry standard deviations:

in $\Delta V: 0.023^{\mathrm{m}}$, in $\Delta(U-B)$ and $\Delta(B-V): 0.015^{\mathrm{m}}$.

The final photometric results are given in Table 1 . This table provides two lines per object, for primary component (A) and for secondary (B). As a primary identifier we choose the IDS-style designation formed from the coordinates for epoch 2000 taken from WDS (1996). The columns give this WDS and Durchmusterung designations $^{2}$ of the star, component designation, $V$,

\footnotetext{
${ }^{1}$ See the tables of extinction coefficients at the La Silla observatory at http://obswww.ls.eso.org/lasilla/atm_ext/extinction.html

${ }^{2}$ In WDS catalogue DM identifiers are taken from Bonn, Cordoba and Cape Durchmusterungs according to declination zones $[+89,-22],[-23,-51]$ and $[-52,-89]$, respectively.
} 

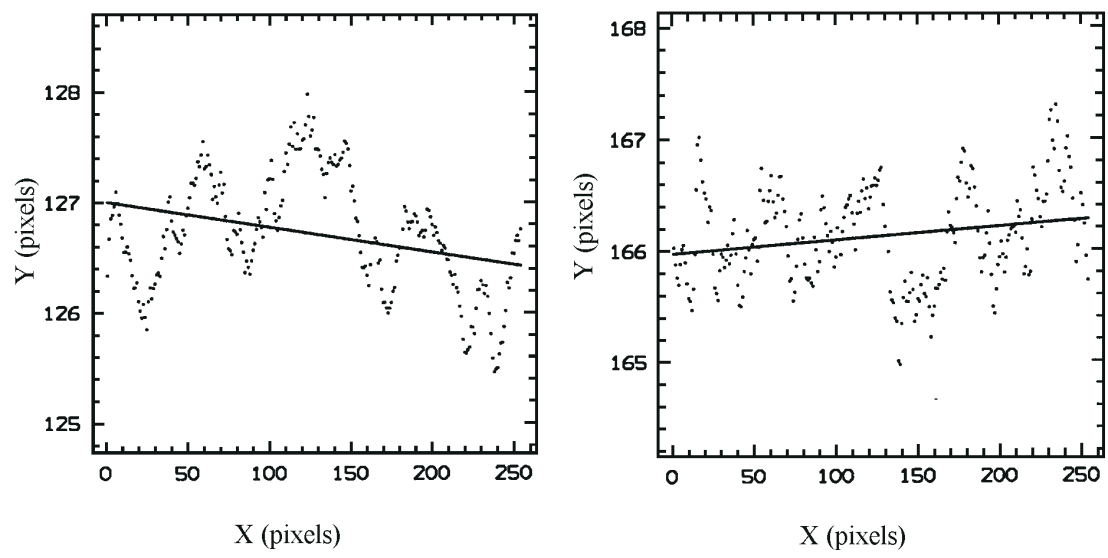

Fig. 1. Two cases of stellar trails and their regression. These trails were taken in the same night but gave quite different apparent inclinations: -0.13 and $+0.06$
$(U-B)$ and $(B-V)$ values with their errors, number of observations $N_{\text {obs }}$ and a note sign. The notes are collected separately in Table 2. The errors were estimated from the individual spread of values in each observation and differences of values obtained in two nights. They do not include transformation errors, which were discussed above, and extinction uncertainties. It should be stressed that as photometry error for the secondaries we give those of differences of their $V$ and colours with primaries. But, since the primary magnitudes were obtained from the total object magnitude $\operatorname{Mag}_{\mathrm{A}+\mathrm{B}}$ and magnitude difference $\Delta$ Mag values, the errors for the A-component include both errors $\epsilon \operatorname{Mag}_{\mathrm{A}+\mathrm{B}}$ and $\epsilon \Delta$ Mag.

\section{Astrometric calibration}

\section{1. $\rho$ and $\theta$ calibration}

Several wide double stars for calibration of differential astrometry parameters $\rho$ and $\theta$ were initially selected from the list of Brosche \& Sinachopoulos (1988). In the pre-Hipparcos era this source was believed to be a comprehensive and reliable compilation of visual binary star parameters. At the time of the present study the Hipparcos data (ESA 1997) became available for some of them. Only two of five pair-calibrators have independent observations of both components in the output catalogue: WDS 09233+0330 = HIP 46028/9 and WDS $12151+0959=$ HIP 59739/7. Their parameters agree with Hipparcos rather satisfactory: differences in separation are 0. . 03 and $-0^{\prime \prime} .06$ and in $\theta$ are $0.28^{\circ}$ and $0.18^{\circ}$, respectively.

The resulting data on our calibrators are shown in Table 4 with the reference to the source finally used.

Averaging of results gave a scale factor of our optical system $S=0.4456 \pm 0.0005$ ("/ pixel) (see below Sect. 4.3). The correction for differential refraction which affects usually the measurements of wide double stars did not introduce significant change in the calibration. The moderate variations of ambient temperature also prevented a substantial scale drift.
Table 4. Astrometric standards' parameters. Reference column: "HIP" = $($ ESA 1997),$" C D S "=($ Brosche \& Sinachopoulos 1988)

\begin{tabular}{llllll}
\hline WDS & $\rho$ & $\epsilon \rho$ & $\theta$ & $\epsilon \theta$ & Ref. \\
\hline $09233+0330$ & $21^{\prime \prime} .25$ & $0^{\prime \prime} 03$ & $311^{\circ} 43$ & 0.06 & HIP \\
$05549+0702$ & $18^{\prime \prime} .62$ & $0^{\prime \prime} 04$ & $73^{\circ} .04$ & 0.10 & CDS \\
$12151+0959$ & $25^{\prime \prime} 95$ & $0^{\prime \prime} 02$ & 244.95 & 0.03 & HIP \\
$22282+1716$ & $8^{\prime \prime} .99$ & $0^{\prime \prime} 03$ & 114.51 & 0.10 & CDS \\
$01150-5538$ & $13^{\prime \prime} 99$ & $0^{\prime \prime} 03$ & $56^{\circ} .22$ & 0.10 & CDS \\
\hline
\end{tabular}

The epochs of Hipparcos, the CDS list of Brosche \& Sinachopoulos (1988) and our average epoch of observations are almost the same, so we needed no corrections for precession or relative proper motion of the components for position angle calibration. The astrometric standard position measurements provided the zero point of the position angle with relatively large uncertainty: $\alpha_{\theta}=\theta_{0}-\theta=89.88 \pm 0$. 16 .

Such a low quality of astrometric calibration data is supposedly related to the very short exposures taken. Indeed, the calibrators were bright objects and were observed in the $V$-band, so the duration of exposures was about $3 \mathrm{~s}$. It is well known (e.g. Lindegren 1980) that it is not possible to achieve good and reproducible astrometric results with such exposures.

We also applied the trail technique for the determination of right ascension direction. Through the observational sets the stellar trails were taken in the $V$-band in total nine times, at least once in a mission. The time of passage of an equatorial star across the field of view was $\approx 10 \mathrm{~s}$. Two illustrative cases of these trails and their linear regression are shown in Fig. 1. It is clear that for trails as well as for astrometric standards a much longer exposure time should be used to get a more accurate calibration.

Evidently, low-frequency modulation of the atmospheric refractive index of line-of-sight air does not allow for a high-accuracy calibration of the position angle with such short exposures. The formal averaging of our 9 values for the inclination of the trail gave a $\theta$ zero point of $89^{\circ} 80 \pm 0^{\circ} 20$. This does not differ from the value obtained 
from the astrometric standard stars reduction. Therefore, as a final value we adopted $\alpha_{\theta}=89.84 \pm 0.1$.

\subsection{Account for a differential chromatic refraction}

We consider here the effect that the different colours of stars result in not equal shifts of their apparent positions. For this differential chromatic refraction (DCR) the separation between the components plays no role, but the spectral difference does (e.g. Pravdo \& Shaklan 1996). The reason is the atmospheric dispersion (while for differential monochromatic refraction it is the increasing of the refraction angle with zenith distance between two stars). Hence, even if we deal with relatively close binaries, we should investigate the significance of the DCR effect.

To check the importance of this effect in our case we calculated its approximate value for all the objects in the following way. First, we computed effective wavelengths in the $U B V$ system for all the classes of stars. The spectral energy distributions $S(\lambda)$ (Straižys \& Sviderskiene 1977) were taken from A. Mironov (Sternberg Institute), who kindly supplied us with ready-to-use electronic tables. Then we computed the effective wavelengths $\lambda_{\text {eff }}$ of stars in $U, B$ and $V$ bands according to the conventional formula (see, for example, Straižys 1977, p. 16). Afterwards, given the known dependence of the refractive coefficient of air $N_{\text {air }}(\lambda)$ (Allen 1977), we computed for each observed star the value of the DCR effect and the apparent displacement of a secondary component relative to a primary. For this we assumed the stars to belong to the Main Sequence (MS) of the $\mathrm{H}-\mathrm{R}$ diagram.

The most significant effect, up to 50 milliarcsec (mas) was found in the $B$-filter; in $U$ and $V$ it was only up to 10 mas; $10 \%$ of values exceed 20,9 and 6 mas in $B, U$ and $V$, respectively. Fortunately, in the $B$ band there is no large difference in $\lambda_{\text {eff }}$ between giant and dwarf stars and the treatment of all the stars as the MS ones does not introduce a large error.

Although the values are relatively small, we applied these corrections to our measurements. The resulting astrometric parameters are given in Table 3. One line per object is given representing the WDS designation and the HIP-number (when available), $\rho$ and $\theta$ with errors and the number of observations. In this table we already averaged the results in different filters; the errors are assigned to the least error among the individual filter values (in most cases - the values in $U$-filter, which was exposed longer). These final errors of the parameters include those of scale factor and $\theta$ zero point. The average uncertainty of our separations $\epsilon_{\rho}$ is less than 10 mas.

\subsection{Comparison with Hipparcos}

The Hipparcos catalogue provides in the Double and Multiple Stars Annex (DMSA) the information for re- solved binaries that is known to have quite a good precision. We compare the $\rho$ and $\theta$ from Hipparcos with our values where it is possible. The cross-identification of our sample of double stars with the Hipparcos stars was performed on the basis of coordinates comparison. The resulting list of common pairs contains 36 objects (see Table 3 , Col. 2); all of them belong to part C of the DMSA (component solutions) and have solution quality $\mathrm{A}$.

First, we find that our position angles differ from the Hipparcos ones by +0.09 on average, with a rms difference of 0.16 . Thus we can conclude that the two sets of $\theta$ values practically do not differ.

Second, the average ratio of Hipparcos separations to ours

$\left\langle\rho_{\mathrm{HIP}} / \rho\right\rangle=1.0020 \pm 0.0002$.

The difference of this value from unity is significant. But taking this factor into account, the derived rescaled $\rho$ in our sample have standard deviation of only 7 mas from the Hipparcos ones, slightly more than the average Hipparcos error of separations for a given sample $\left(\left\langle\epsilon_{\rho}^{\mathrm{HIP}}\right\rangle=5.1 \mathrm{mas}\right)$.

\section{Tertiary components}

On some object frames there were one or several faint stars located close to the main pair. To make our information on the environment of our doubles more comprehensive we tried to detect all the stars lying closer than $\approx 15-20^{\prime \prime}$ to the primary. Also some relatively bright stars were sometimes measured at larger separations. Too faint, tertiary components were not measured in the ultraviolet.

Some fifty tertiaries were found, with a $V$-magnitude difference with the primary up to $7^{\mathrm{m}}$. At the level of $\Delta V \approx 5^{\mathrm{m}}-6^{\mathrm{m}}$ the scope is apparently complete. This detection was followed by an immediate ("on-fly") estimation of the $V$-and $B$-magnitude differences with the primary component, obviously a rather rough estimate because normally the magnitude difference is too large: errors are about $0.2^{\mathrm{m}}$ in $\Delta V$ and $0.3^{\mathrm{m}}$ in $\Delta(B-V)$. Relative positions of detected stars have an uncertainty of about $1-2$ pixels $\left(0^{\prime \prime} .5-1^{\prime \prime}\right)$.

The parameters of all surrounding stars are collected in Table 5. Columns give the WDS identifier, a companion identifier, differential photometry data $\Delta V$ and $\Delta(B-V)$ and position parameters $\rho$ and $\theta$ relative to the primary. The last column denotes the reference to the method with which these parameters were obtained. Original "on-fly" data are signed as "F", while other cases are described below.

For some "tertiaries" we made an attempt to improve the quality of our data.

The results from full-aperture $\left(\approx 34^{\prime \prime}\right)$ photometry of sufficiently distant and not very faint star are presented with a reference "P". They were obtained by exactly the same technique as the total object photometry. The astrometry quality remains the same as for " $F$ "-cases, while 
Table 5. Faint stars in the vicinity of the objects. Low-case identifiers ID are arbitrary ones. See more description in text

WDS ID $\Delta V \Delta(B-V)$ rho $^{\prime \prime}$ theta $^{\circ}$ Refs.

\begin{tabular}{|c|c|c|c|c|c|c|}
\hline $05314-0206$ & $\bar{d}$ & 6.5 & 0.4 & 11.2 & 85 & $\overline{F P}$ \\
\hline $05500-3612$ & $\mathrm{C}$ & 3.4 & 0.4 & 8.3 & 324 & $\mathrm{~F}$ \\
\hline $06049-0243$ & $\mathrm{c}$ & 5.2 & 1.1 & 14.5 & 247 & $\mathrm{FP}$ \\
\hline $06321+0458$ & $\mathrm{c}$ & 7.0 & 0.1 & 12.8 & 12 & $\mathrm{~F}$ \\
\hline $06420-0446$ & $\mathrm{c}$ & 6.0 & 1.5 & 8.9 & 177 & $\mathrm{~F}$ \\
\hline $07035-0828$ & d & 4.5 & 0.8 & 17.5 & 19.0 & PFD \\
\hline 07086-1359 & $\mathrm{C}$ & 3.3 & 0.8 & 13.0 & 354 & $\mathrm{~F}$ \\
\hline $07165-1559$ & $\mathrm{c}$ & 6.2 & 0.6 & 15.5 & 160 & $\mathrm{PF}$ \\
\hline $07198+1322$ & $\mathrm{c}$ & 6.0 & 0.2 & 14.8 & 331 & $\mathrm{~F}$ \\
\hline \multirow[t]{2}{*}{$07222-2412$} & $\mathrm{c}$ & 4.6 & 0.2 & 5.4 & 305 & $\mathrm{~F}$ \\
\hline & d & 5.1 & 0.3 & 11.6 & 344 & $\mathrm{~F}$ \\
\hline $07457-1720$ & $\mathrm{c}$ & 6.1 & - & 4.8 & 227 & $\mathrm{~d}$ \\
\hline $07597-4429$ & $\mathrm{c}$ & 3.1 & 0.8 & 23.9 & 286.9 & $\mathrm{D}$ \\
\hline $08148-2728$ & $\mathrm{c}$ & 5.6 & 0.5 & 14.6 & 348 & $\mathrm{~F}$ \\
\hline \multirow[t]{3}{*}{$08450-5821$} & $\mathrm{c}$ & 5.0 & 1.0 & 17.2 & 212.0 & DP \\
\hline & d & 3.1 & 1.0 & 33.1 & 181.7 & $\mathrm{D}$ \\
\hline & e & 0.7 & 1.2 & 42.0 & 166.3 & $\mathrm{D}$ \\
\hline \multirow[t]{2}{*}{$09080-4615$} & $\mathrm{c}$ & 5.7 & 1.2 & 12.4 & 212.3 & $\mathrm{~d}$ \\
\hline & d & 1.1 & 1.7 & 24.9 & 68.3 & d \\
\hline $09259-5354$ & $\mathrm{c}$ & 5.8 & 1.4 & 11.7 & 46 & $\mathrm{~F}$ \\
\hline $09516-4231$ & $\mathrm{c}$ & 6.4 & 0.0 & 21.6 & 122 & $\mathrm{~F}$ \\
\hline \multirow[t]{2}{*}{$10354-5347$} & $\mathrm{c}$ & 5.0 & 1.0 & 8.4 & 238 & $\mathrm{~F}$ \\
\hline & d & 3.8 & 0.4 & 13.9 & 116 & $\mathrm{~F}$ \\
\hline $10458-5719$ & $\mathrm{c}$ & 4.4 & 1.6 & 16.5 & 63 & $\mathrm{~F}$ \\
\hline $10490-4348$ & $\mathrm{c}$ & 6.7 & 0.3 & 20.3 & 83.5 & d \\
\hline $11006-7259$ & $\mathrm{c}$ & 5.7 & 0.8 & 5.8 & 85 & $\mathrm{~F}$ \\
\hline $11017-5716$ & $\mathrm{c}$ & 6.9 & 0.8 & 11.3 & 117.3 & d \\
\hline $11202-5625$ & $\mathrm{c}$ & 5.7 & 0.7 & 12.0 & 110 & FP \\
\hline \multirow[t]{2}{*}{$11290-6334$} & $\mathrm{c}$ & 2.5 & 0.4 & 26.8 & 184.0 & $\mathrm{D}$ \\
\hline & d & 5.1 & 0.8 & 30.2 & 178.0 & DP \\
\hline $11363-4759$ & $\mathrm{c}$ & 7.1 & 0.5 & 11.8 & 307 & $\mathrm{~F}$ \\
\hline $11426-5136$ & $\mathrm{c}$ & 3.9 & 1.6 & 50.3 & 242 & $\mathrm{P}$ \\
\hline $12231-6405$ & $\mathrm{c}$ & 5.1 & 0.4 & 9.3 & 149 & d \\
\hline $13080-5641$ & $\mathrm{c}$ & 5.5 & 0.8 & 24.1 & 152 & $\mathrm{P}$ \\
\hline $13435-3739$ & $\mathrm{c}$ & 6.5 & 0.3 & 20.5 & 222 & $\mathrm{~F}$ \\
\hline $13583-6538$ & $\mathrm{c}$ & 6.1 & 0.3 & 9.8 & 39 & $\mathrm{~F}$ \\
\hline $14405-5833$ & $\mathrm{c}$ & 2.8 & 1.2 & 42.6 & 207.8 & d \\
\hline \multirow[t]{2}{*}{$15581-6545$} & $\mathrm{c}$ & 5.5 & 1.0 & 9.5 & 202 & d \\
\hline & d & 4.7 & 0.0 & 26.0 & 339.2 & d \\
\hline \multirow[t]{2}{*}{$17232-3946$} & $\mathrm{c}$ & 5.3 & - & 13.2 & 264.5 & d \\
\hline & d & 4.3 & 0.5 & 23.9 & 292.4 & d \\
\hline \multirow[t]{5}{*}{$17357-4731$} & $\mathrm{c}$ & 0.7 & 0.1 & 10.7 & 264 & d \\
\hline & d & 3.7 & 0.4 & 7.8 & 189 & d \\
\hline & e & 5.6 & 0.6 & 12.9 & 181.4 & $\mathrm{~d}$ \\
\hline & $\mathrm{f}$ & 5.1 & 0.4 & 13.6 & 48.7 & d \\
\hline & g & 4.1 & 0.5 & 28.0 & 55.7 & d \\
\hline $18135-4737$ & $\mathrm{c}$ & 4.9 & 0.8 & 4.2 & 117 & d \\
\hline $19146-3615$ & $\mathrm{c}$ & 5.5 & 0.6 & 49.7 & 330 & $\mathrm{P}$ \\
\hline $20573-2138$ & $\mathrm{c}$ & 4.9 & 0.6 & 49.0 & 274 & $\mathrm{P}$ \\
\hline \multirow[t]{4}{*}{$21124+1432$} & $\mathrm{C}$ & 4.0 & 0.9 & 36.6 & 228 & $\mathrm{P}$ \\
\hline & $\mathrm{D}$ & 4.0 & 1.0 & 38.3 & 245 & $\mathrm{P}$ \\
\hline & e & 5.5 & 0.7 & 19.5 & 10 & $\mathrm{~F}$ \\
\hline & $\mathrm{f}$ & 6.0 & - & 37.0 & 144 & $\mathrm{~F}$ \\
\hline \multirow[t]{2}{*}{$21293-1720$} & $\mathrm{c}$ & 4.1 & 0.4 & 36.0 & 326 & $\mathrm{P}$ \\
\hline & d & 5.1 & 0.7 & 27.6 & 329 & $\mathrm{P}$ \\
\hline
\end{tabular}

errors of $V$ and $(B-V)$ are less, about $\pm 0.1^{\mathrm{m}}$ and $\pm 0.15^{\mathrm{m}}$, respectively.

Some multiple stars with up to 5 visual companions were measured with the help of DAOPHOT. These results are added to Table 5 with a remark "D" or "d", depending upon the choice of stars for the point spread function model. The first case corresponds to the usage of a bright enough and well separated star in the field. The "d"-method corresponds to the use of an artificial PSF star with Moffat shape determined from the binary star fit (see Sect. 2). The estimated precision of the DAOPHOT technique is $\pm 0.05^{\mathrm{m}}$ in photometry and $\pm 0.1^{\prime \prime}$ in astrometry.

All the errors of photometry given above correspond to magnitude difference $<5^{\mathrm{m}}$; they are about 1.5 times larger for $\Delta V$ between $5^{\mathrm{m}}$ and $6^{\mathrm{m}}$ and 2 times larger for fainter stars.

To make sure that we have not assigned the wrong identifier to a previously known tertiary component, we extracted the existing information on them from WDS (1996). The tertiaries were identified using their $\rho$ and $\theta$. WDS gave four identifications of our tertiaries, their identifiers in Table 5 are given in upper-case; all the rest are in lower-case letters.

Evidently, most stars are field ones. The comparison of their photometric parallaxes to spectroscopic ones of primaries (or to those from Hipparcos when available) is the subject of further study in order to decide about the physicity of some companions to the main pairs $\mathrm{A}+\mathrm{B}$. An interesting case is a non-WDS bright and close tertiary companion "c" of WDS 17357-4731 = CP-47 11654. According to its photometry, it has a higher probability to be a physical one than the B-component.

\section{Conclusion and discussion}

The topics discussed above concern the reduction technique and our data set from the observational side. Further interpretation, like in-depth determination of the physical status of components, interstellar reddening account, recognition of evolutionary effects etc., exceeds the scope of this paper. We present some conclusions directly related to the observations.

- We find that in most cases the secondary components are also early-type A-F stars $\left(60 \%\right.$ of $(B-V)_{B}<0.3$; $\left.93 \%:(B-V)_{B}<0.58\right)$. This means that we deal with mostly physical binaries rather than optical pairs.

Figure 2 shows $\Delta V$ versus $\Delta(B-V)$ between the $\mathrm{A}$ and $\mathrm{B}$ components. The symbol type corresponds to the primary component luminosity class, taken from the WDS (1996) spectral class. Squares are the MS stars, filled triangles - giants and subgiants, open triangles - with unknown luminosity class and "stars" are peculiar objects. Left and right dashed lines represent the inclinations of the MS for A0 and F0 stars, respectively. Many secondaries fall inside or around the 


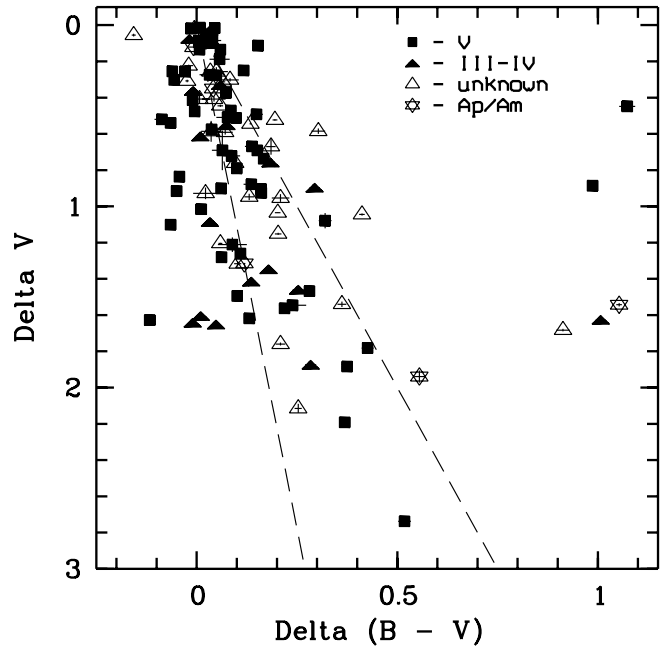

Fig. 2. $\Delta V-\Delta(B-V)$ diagram. The two lines delimit an area corresponding to the MS for A0-F0 stars (see text)

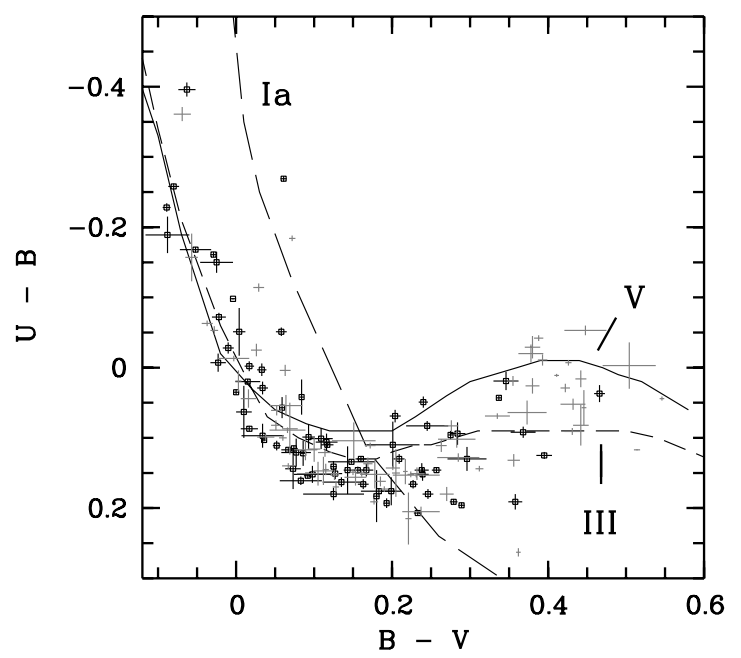

Fig. 3. $(U-B)-(B-V)$ diagram: all the primaries (dark heavy crosses) and all but one (WDS 19146-3615 B) secondaries (light crosses) are shown. Solid, short- and long-dashed lines represent the dwarfs, giants and supergiants, respectively (taken from Straižys 1977)

area between these two lines; the outliers may represent the optical components, the natural width of the MS, or evolutionary effects. Further comparison of our astrometric data to old measurements will allow us to reject optical pairs, checking the consistency of photometric or spectroscopic parallaxes with hypothetical dynamical ones (Russel \& Moore 1940).

- A large part of our objects have both $(U-B)$ and $(B-V)$ indices of the components measured. For these 75 stars we can construct the two colour diagram (Fig. 3) for primaries and secondaries. One needs to correct the data for interstellar absorption before giving any astrophysical interpretation since our objects are relatively distant ones: the distance modulus $m-M$ varies from $6^{\mathrm{m}}$ to $11^{\mathrm{m}}$. It can be seen that our stars occupy the thick area between the MS and evolved stars of luminosity classes III-Ia. But, since we do not know so far the reddening factor of our objects we cannot separate the effects of evolution or chemical composition from those of interstellar absorption.

- A lot of faint stars are discovered in a close neighbourhood of our objects; some of them have separations to the primary similar to those in the main pair A+B. Very few of these cases are possible physical multiples. Although trapezium-like configurations are detected among a number of OB-stars (like in the Orion nebula), they must have been already disrupted in substantially older systems of spectral class A (e.g. Eggleton \& Kiseleva 1995). So, further investigations of our data are required in this field. The existence of low-mass distant companions in A-type multiples is also of great interest.

- The comparison with Hipparcos data reveals the high degree of similarity of our data to those of Hipparcos when available. This proves our astrometric information as good and reliable for investigation of relative motion of A-type double star components.

Acknowledgements. We would like to thank Dr. A. Tokovinin (Sternberg Institute) for discussions of the current work and paper. In the final steps the data from Astronomical Data Center were extensively used.

This work was supported by the OSTC/DWTC of Belgium through a Belgo-Russian Bilateral Cooperation program (N.S.), the project "Pôles d'Attraction Interuniversitaires" P04/05 initiated and financed by the Belgian Federal Scientific Services (DWTC/SSTC) (D.S.); and an IAU traveling grant (N.S.).

\section{References}

Allen C.W., 1973, Astrophysical quantities. London: Univ. of London, Athlone Press, 3rd edition

Brosche P., Sinachopoulos D., 1988, Bull. Inform. CDS No. 36 Burki G., Rufener F., Burnet M., et al., 1995, Messenger 80, 34

Eggleton P., Kiseleva L., 1995, ApJ 455, 640

European Space Agency, 1997, The Hipparcos and Tycho Catalogues, European Space Agency, SP-1200, Paris: ESA

Graham J.A., 1982, PASP 94, 244

Grenon M., 1992 (private communication)

Landolt A.U., 1973, AJ 78, 959

Lindegren L., 1980, A\&A 89, 41

Pravdo S.H., Shaklan S.B., 1996, ApJ 465, 264

Press W.H., Flannery B.P., Teukolsky S.A., et al., 1987, Numerical Recipes. The Art of Scientific Computing. Cambridge University Press, New York

Russel H.N., Moore C.E., 1940, Masses of Stars. Univ. Chicago Straižys V., 1977, Multicolor Stellar Photometry, Mokslas Publishers, Vilnius

Straižys V., Sviderskiene Z., 1972, Bull. Vilnius Obs. 25, 3

Worley C.E., Douglass G.G., 1984, 1996, The Washington Visual Double Star Catalog. US Naval Observatory (WDS) 